\title{
Is there evolution in the black hole - bulge relation?
}

\author{
Andreas Schulze ${ }^{1}$ and Lutz Wisotzki ${ }^{2}$ \\ ${ }^{1}$ Kavli Institute for Astronomy and Astrophysics, Peking University, 100871 Beijing, China \\ email: aschulze@pku.edu.cn \\ ${ }^{2}$ Leibniz-Institut für Astrophysik Potsdam (AIP), An der Sternwarte 16, \\ 14482 Potsdam, Germany
}

\begin{abstract}
We present a framework to investigate sample selection effects on the observed $\mathrm{BH}$ - bulge relation and its evolution with redshift. We particularly discuss an active fraction bias and a luminosity bias. Applying our framework to literature studies, we cannot find statistically significant evidence for an evolving BH-bulge relation.
\end{abstract}

Keywords. Galaxies: active - Galaxies: nuclei - quasars: general

The existence of the observed black hole - bulge relation implies a close link between the growth of black holes and the evolution of galaxies. The redshift evolution of this correlation provides constraints on the origin of this link.

In Schulze \& Wisotzki (2011) we presented an investigation of sample selection effects that influence the observed black hole - bulge relation and its evolution with redshift. Our framework can be used to investigate all kinds of selection effects on the black holebulge relation, but we focus on the consequences of using broad-line AGN and their host galaxies to search for evolution in the black hole - bulge relation.

We particularly consider two important effects. (i) The active fraction among SMBH locally decreases with increasing black hole mass (Schulze \& Wisotzki 2010). In connection with intrinsic scatter of the black hole - bulge relation this effect implies a bias towards a low BH mass at given bulge property. (ii) The flux limited nature of observational AGN samples leads to a bias towards a high black hole mass at given bulge property, as already discussed by others (Salviander et al. 2007, Lauer et al. 2007).

A quantitative prediction of these biases requires (i) a realistic model of the sample selection function, and (ii) knowledge of the relevant underlying distribution functions. At higher redshift, we have currently limited observational constraints on the latter.

Using the available constraints, we estimate the expected magnitude of sample selection biases for a number of recent observational attempts to study the BH-bulge evolution (e.g. Salviander et al. 2007, Merloni et al. 2010). In no case do we find statistically significant evidence for an evolving BH-bulge relation. While the observed apparent offsets in the $\mathrm{BH}$-bulge ratio from the local relation can be quite large, the sample selection bias estimated from our formalism is typically of the same magnitude.

\section{References}

Lauer, T. R., Tremaine, S., Richstone, D., \& Faber, S. M. 2007, ApJ, 670, 249

Merloni, A., Bongiorno, A., Bolzonella, M., et al. 2010, ApJ, 708, 137

Salviander, S., Shields, G. A., Gebhardt, K., \& Bonning, E. W. 2007, ApJ, 662, 131

Schulze, A. \& Wisotzki, L. 2010, A\&A, 516, A87+

Schulze, A. \& Wisotzki, L. 2011, A\&A A, 535, A87 